\title{
Process Signatures as a Measure for Loopless Production
}

\author{
Tong Zhao', Frank Vollertsen ${ }^{1,2}$ \\ ${ }^{1}$ BIAS-Bremer Institut für angewandte Strahltechnik GmbH, Bremen, Germany \\ ${ }^{2}$ University of Bremen, Faculty of Production Engineering-Mechanical Engineering \& Process Engineering, Bremen, Germany \\ Email:mikrostrukturierung@bias.de
}

How to cite this paper: Zhao, T. and Vollertsen, F. (2017) Process Signatures as a Measure for Loopless Production. Engineering, 9, 328-337.

https://doi.org/10.4236/eng.2017.93017

Received: March 3, 2017

Accepted: March 28, 2017

Published: March 31, 2017

Copyright (C) 2017 by authors and Scientific Research Publishing Inc. This work is licensed under the Creative Commons Attribution International License (CC BY 4.0).

http://creativecommons.org/licenses/by/4.0/

\begin{abstract}
Using control and repairing loops to remove production errors is not the only solution to increase the manufacturing yield. The production of errors can also be directly avoided, prevented or eliminated, even as early as in the planning phase. This paper suggests that the idea of Process Signatures can help to achieve Loopless Production. Loopless Production offers an option to guarantee the production quality towards the vision of the zero-defect manufacturing. It is considered that closed loops are used in a production process chain to identify and to correct the unknown and the systematic errors. These errors can actually be avoided through specifically adjusted or optimal arranged production processes. This puts a higher demand on the understanding of processes, which involve various energy-material interactions. This demand can be met via Process Signatures which aims to develop a process-independent description method of effects of processes. A supportive relationship is foreseen between Process Signatures and Loopless Production. The combination of these two ideas shall allow the simplification of the work for the rationalization of process sequences, the streamlining of closed loops as well as the selection of optimal substitute processes.
\end{abstract}

\section{Keywords}

Unknown Error, Systematic Error, Process Chain, Closed Loop, Energy-Material Interaction

\section{Introduction}

Material needs of humans stimulate the development of manufacturing industries, whereby epochal production philosophies are generated during the process of the improvement of the instrumental rationality. As in concrete industrial activities, meeting the demand of improvements is turned into a common value of 
technical promotion of specific fields. The methodologies of the production philosophies address approaches to satisfying the demand.

With the advances in science and technology, the production of fully-functional parts instead of improving given geometric tolerances gains increasingly attention [1]. For processing industry, the selection process and the parameter study are conventionally based on the trail-and-error paradigm and on fixed outputs. This causes a low efficiency and thus a high cost of the preparatory experiments. For new production types, such as the mass customization manufacturing or the decentralized manufacturing, the requirement of flexible outputs cannot be met through the traditional way.

In parallel, the industry is also developing in pursuit of a zero-defect manufacturing. Towards this vision, well-known quality management systems, such as the Lean Management which focuses on reducing wastes or the Six Sigma which focuses on reducing deviations, have been widely proved to be effective. Besides, new emerging approaches have been suggested, such as the EU-funded MEGaFiT-project [2] and IFaCOM-project [3] which treats this issue with the concentration on the adaptive process control and the self-optimizing process control, respectively.

\section{Process Signatures}

Generally, a process window is described by using a combination of process-specific parameters, like "scanning speed", "cutting speed", "discharge duration" or "pulse duration". Such parameters make the description of a particular process intuitive and its application operable. However, material properties are essentially altered by the change of the whole process condition. Different processes might somehow have an overlap between their total effects. A same process condition might thus be created in different ways. The other values which are usually regarded as constant, like the chemical compositions of materials, would also become parameters of certain processes, if they could be set separately during the process operation. All these constrain define the effect of a process which leads to a functionalization of material. If e.g. two parts, which were processed through different ways, both could meet a certain functional requirement, there should be something common that has happened both in these two processes. How to translate the effects of different processes into some common intermediate steps is still an open question.

In order to solve this problem, an attempt at developing a process-condition independent method has been made for the description of topography-related changes in thermal-mechanical processes [4]. For more general cases, the academia seeks for ways to directly predict the required processing procedures for desired functional properties of modified surfaces [5]. It is believed that processes are essentially determined by different kinds of interactions between materials and combinations of mechanical, thermal and/or chemical energy [6]. This idea, which is named as "Process Signatures" [5], indicates a potential possibility to connect the material changes induced by processes with the basic natures of energy inputs and the following conversions, as shown in Figure 1. 


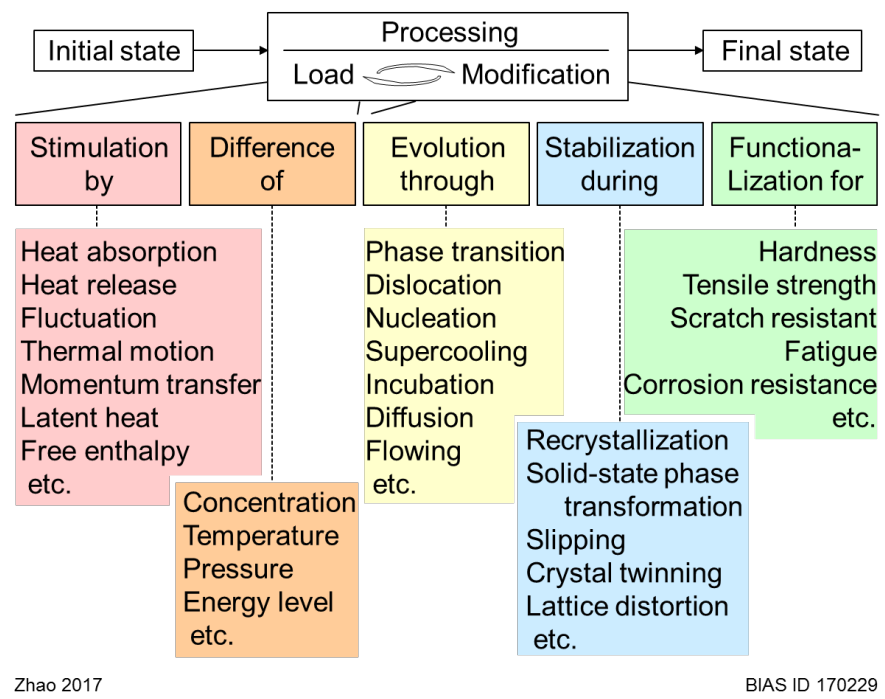

Figure 1. Schematic of process signatures (based on [6]).

A process, which changes the material from an initial state to a final state, can be regarded as an imagined cause-effect chain within a control volume. A possible forward chaining is that some differences can be stimulated in material and cause evolutionary changes. The changes initiate the next stable state with characterizable functional properties. For desired functional properties, inversely, the corresponding evolutionary changes must be achieved which are caused at the very beginning by the appropriate stimulations. Besides the difficulty in building such a chain, the detailed analysis involves more difficult issues e.g. that not all of the properties will be formed at the same pace during a process because of their different cumulative responses to the energy input [7].

\section{Loopless Production}

For a practical application, the decision making of choosing appropriate processes can benefit from the precise description of the cause-effect chain. However, being able to describe all the desired details of a process does not mean being able to realize those details, especially economically. On one hand, the initial state of material in one process is always the final state left by the previous processes. In the case of e.g. flexible manufacturing, such an initial state varies and is not always in the ideal or the familiar state for conducting one given process. On the other hand, even if such a cross-process description for an existed process chain could be achieved, it still does not mean that the process chain itself is impeccable or for targeted outputs is an irreplaceable option.

The detailed categorization of the effects and side effects of the existed processes is just an added measure of increasing the yield in manufacturing. The advent of new processes driven by the progress of science and technology provides more options for achieving the targeted outputs. A process could be optimized, but only with a limited potential, through the exploit of the old tool [8]. The improvement of the tool itself makes actually significant advantages, e.g. the replacement of the multiple patterning by the EUV technology in semiconductor manufacturing [9]. Such a replacement puts high demands on the tool, but it 
removes directly the defect cause and simplifies fundamentally the processing.

However, many efforts still have been put into enhancing stopgaps, like a quality inspection or a repair loop in a problematic process chain, to reduce the rejection rate but not to deal with the root causes [10]. In traditional production, it is considered that every process in a process chain makes its own contribution to changing a given initial state into a final state. Due to improper applications of processes, the foreseeable deviations from the ideal final state could be regarded as "systematic errors". Other unforeseeable deviations caused by the insufficient understanding of the functional mechanism or by the imperfect fulfillment of processes could be regarded as "unknown errors". These errors would be kept in the workpiece and could only be detected by the comparison of the processed workpiece with the reference ideal product. The detected difference will be fed back to optimize the process parameters to control the difference into an allowable tolerance, as shown in Figure 2(a).

Although closed loops could be able to dispose of imperfect articles, the introduction of closed loops itself would bring the process chain more uncertainties and therefore lead to a lower reliability. To deal with this dilemma, an idea named as "Loopless Production" is proposed which emphasizes that the precondition to achieve the zero-defect manufacturing is the achievement of zero-defect process chains [10]. An ideal process chain is that every process only makes an error-free contribution on the workpiece to achieve the final state evolutionarily, as shown in Figure 2(b). If not all of the processes is ideal, the process chain should be organized to be flexible to adopt replacement technologies. This requires that the unknown errors should be restricted within processes or even better within tools.

This paper suggests that a supportive relationship is foreseen between the Process Signatures and the Loopless Production. The combination of these two ideas shall allow the simplification of the work for the rationalization of process

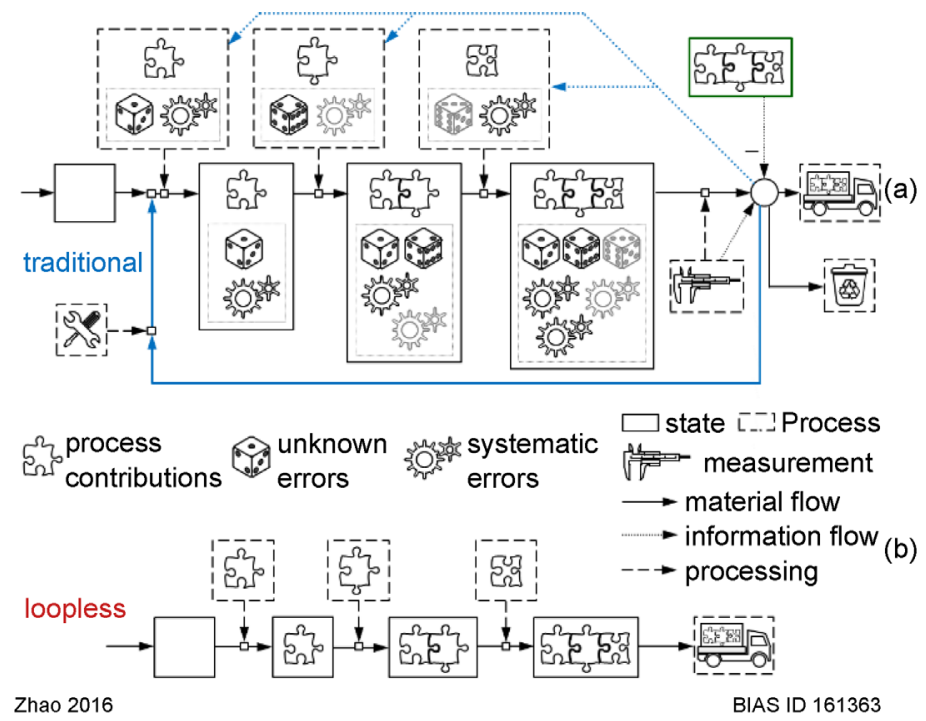

Figure 2. Schematic of traditional process chain and ideal loopless process chain. 
sequences, the streamlining of closed loops as well as the selection of optimal substitute processes. Errors in manufacturing could thus be overcome through rationally selected and coordinated processes rather than just using closed loops.

\section{Approaches to Loopless Production}

Closed loops themselves are remedies for error handling and could help to increase the yield, but they add complexity and bring more uncertainties to a process chain. While increasing the resource consumption, however, closed loops cannot avoid, prevent or transfer the risk of errors. A part of the unknown errors of a process could be turned into systematic errors through a thorough analysis of the mechanism of the energy-material interaction. The systematic errors are foreseeable and thus can be avoided, prevented or transferred. Based on the premise that the root cause of the errors should be solved, the optimization of a process chain needs to focus on the selection of appropriate and the replacement of outdated as well as the coordination of processes. Towards the vision of an zero-defect manufacturing, three approaches, "process control assimilation", "process chain extension" and "process chain substitution", have been suggested [10].

\section{Process control assimilation}

Imaging that there is a scenario in which a process is made up of three mutual independent processes. A workpiece with an initial state will be changed by the processes step by step to approach gradually to the targeted final state. The state will be checked after the third process to decide whether the product can pass the inspection or need to be repaired or disposed. The knowledge of the mechanism of a process and the practical accuracy of the equipment to fulfill a process could be limited. If, therefore, a process cannot be fully mastered, the process will introduce unknown errors into the workpiece while making the useful contribution. In such a setup, if an unknown error occurs in the second process, the error will be brought into and be visible only after the third process, as shown in Figure 3(a). Besides, the correction of the error cannot be precisely conducted; hence it cannot be done in time within one process but needs an interactive adjustment of the three processes. The process chain, therefore, excludes new processes. For the optimization of this kind of process chains, which are mostly still in the run-in period, it must be tried to narrow down the closed loop to restrict the impact of the unknown error, as shown in Figure 3(b).

\section{Process chain extension}

Processes, by their very nature, change the material state with technological characteristics within the capability limits. Ideally, the technological characteristics match the expectation of the product quality. Due to possible improper applications of processes, foreseeable deviations from ideal final state as systematic errors might be caused. In a traditional way with help of closed loops, the process chain can be slightly optimized according to the feedback of the tested consequences of an error, as shown in Figure 4(a). However, since the error is foreseeable, it should be possible to extend such a process chain purposefully by 

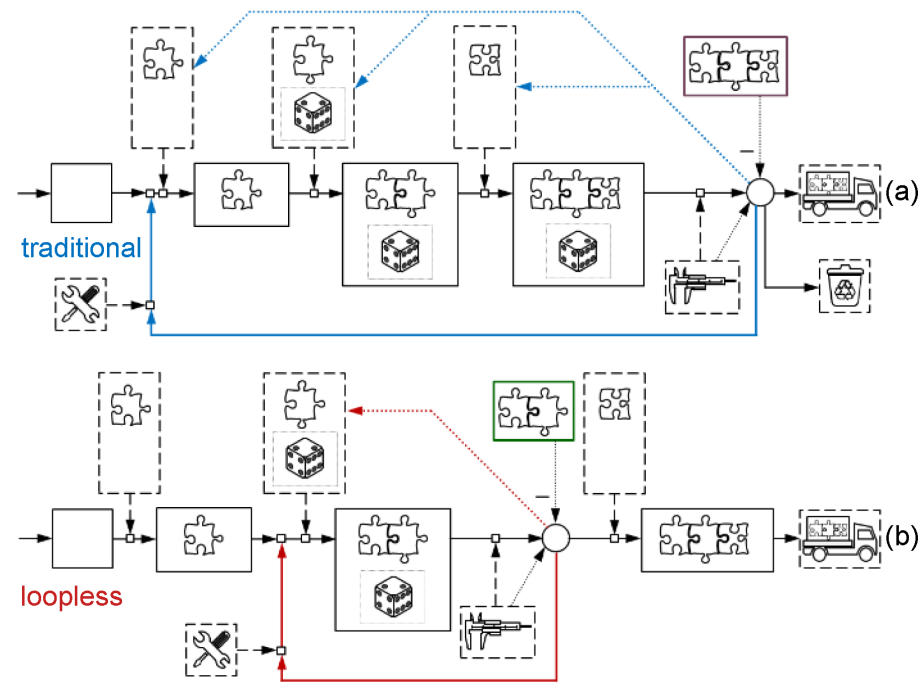

Zhao 2016

BIAS ID 161365

Figure 3. Schematic of process control assimilation.
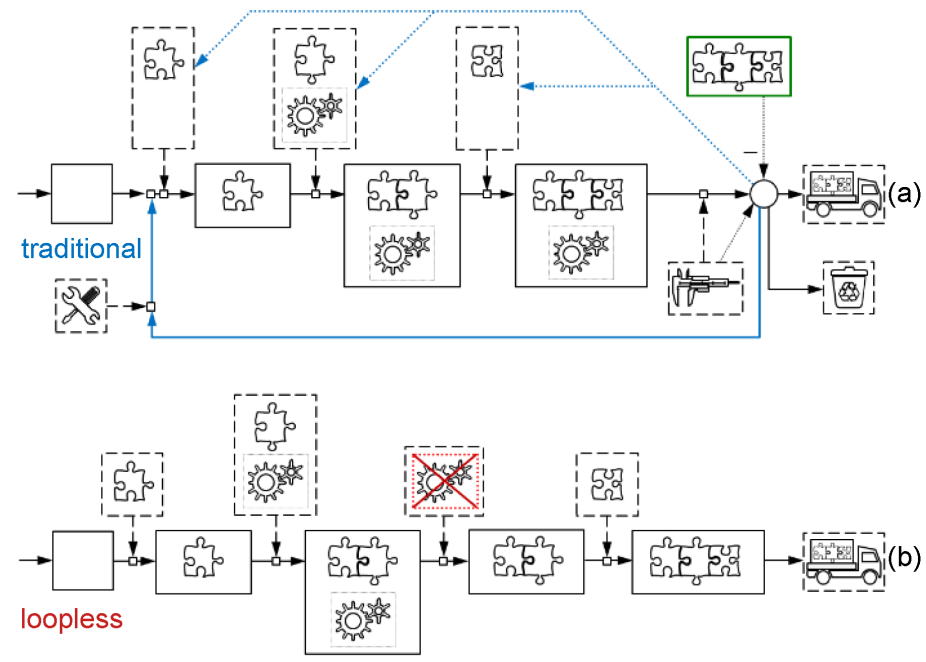

Zhao 2016

BIAS ID 161364

Figure 4. Schematic of process chain extension.

adding a counter process, which is employed just to remove or to prevent the error, as shown in Figure 4(b). The process with the systematic error and the corresponding counter process can be regarded as a whole, which eliminates the error inside and acts error-free outside.

\section{Process substitution}

As mentioned before, the approach "process chain assimilation" aims at narrowing a large closed-loop into a small but precise one to limit the impact of the unknown errors. A part of the unknown errors would be turned into systematic errors, if the process can be fully mastered both on the theoretical side and on the practical side. Even so, as shown in Figure 5(a) that the errors are handled by closed-loops separately, the closed-loops are still redundant due of the additional resource consumption for recouping the losses caused by the errors. This predicament could be postponed through the application of the approach "process 


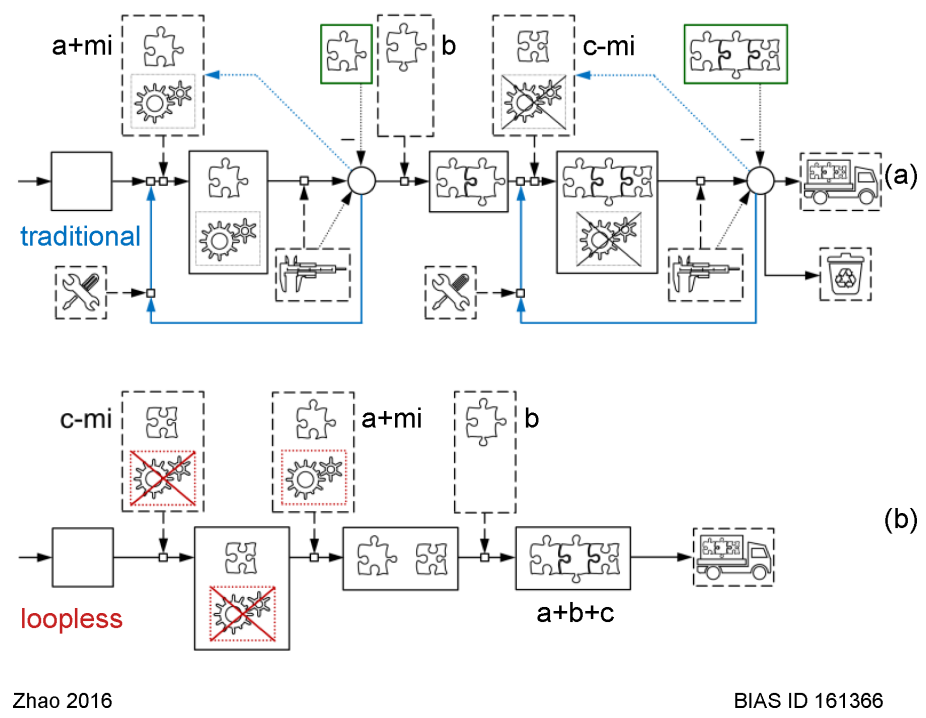

Figure 5. Schematic of process substitution.

chain extension" but still with complication. However, if possible, it would be optimal that the errors of two successive processes could cancel each other out. Thereby, the errors are no longer regarded as negative but interim results, as shown in Figure 5(b).

The effect of a process can be in general terms described a complex number " $x+$ yi" (or a tensor), where " $x$ " and " $y$ " are two real numbers and " $i$ " is the imaginary unit. If the expected process contribution is indicated by the real part, then the error can be indicated by the imaginary part. As shown in Figure 5(a) that e.g. the first process is " $a+$ mi", the second process is " $b+0 \mathrm{i}$ " and the third process is " $c-m i$ ". If the order of the processes could also be changed, then a loopless process chain can be achieved as shown in Figure 5(b), namely $(c-m i)+$ $(a+m i)+b=c+a+b=a+b+c$.

\section{Process Signatures as a Measure}

The realization of Loopless Production requires firstly the customizations of the process chain according to the delivered initial state of workpieces and the targeted delivery product state, and secondly, the exact running performance of the process chain. Such a customization and the exact running should be additionally based on the application of closed loops as limited as possible.

For this, it is necessary to optimize the applied processes or enable a reasonable arrangement of imperfect processes. The following preconditions must be fulfilled that, 1) the effect of the initial state of the workpiece on the result of energy-material interactions is clear; 2) unknown errors are eliminated or turned into systematic errors; 3) systematic errors can be avoided, prevented or transferred through specifically set process orders or new processes; 4) the process chain is flexible to allow changes in terms of the compatibility.

Process Signatures is expected as a measure to deduce a common description method of specific energy-material-interactions of different processes based on a unified theoretical foundation. This is other than a pure empirical and statistical 
method for determining the correlation between the inputs and outputs within a process but explains the correlation in the way of material physics. Regarding the material surface as the boundary, Process Signatures attends to describe a process which is not based on the process variables on the side of the tool but from the perspective of the material. The core issues are that, first, what physics fields will be formed in the material; second, how will the material be evolved by the fields [7].

The answers to the issues facilitate the understanding that how to fulfill the four preconditions for the realization of Loopless Production. Furthermore, if a required process chain must be figured out from the given initial state and the desired final state, Process Signatures contributes to scope that to what extent the process chain can be realized by the existing processes. The uncovered range indicates the development demands of new processes.

\section{Research Emphases}

Process Signatures and Loopless Production point out two different but convergent research directions to optimize the production system towards the zero-defect manufacturing of fully-functional parts. For a synergetic research, following topics are considered important.

The detailed study of the mechanisms of energy-material interactions within the framework of Process Signatures could contribute both the optimization of existing processes and the development of desired new processes. This would help meet the demand of Loopless Production on the selection and the coordination of proper processes for building loopless process chains. It is required that not just a few indicators, e.g. the strength or hardness, but also other associate factors, e.g. unavoidable phase transformation or plastic deformation generated in different ways of strengthening, should be taken into account.

On one hand, however, even if processes can be categorized into several groups according to basic energy forms applied in processes, it does not mean that these energy forms are compatible in any combination. Therefore, it is still unanswered that a combination in which situation and to which extent is feasible to realize a new desired process. Furthermore, even if the loads imposed on the material by the combination can be accurately and precisely initiated, e.g. through a precooled grinding wheel with a certain rotation speed and contact force, question about how to describe the conversion of these inputs during the process until that the workpiece reaches a new steady state is still open. For this, one perspective is that these primary inputs evolve in space and time and initiate their specific successive internal inputs and the corresponding property changes in material [7].

On the other hand, the realization of Loopless Production relies actually both on the technical and the management progress of the whole manufacturing. Desired outputs can be produced not only depends on the error-free conduction of every process but also on the delivery of the input material with a consistent quality. In addition, a possible mistake caused by a subsequent process should 
not be able to ruin all the previous efforts during the course of the fabrication. This should be taken into consideration as early as in the design phase of a product in order to avoid having to use big closed loops. Furthermore, particularly in mass production, a reduction of reproducibility due to human errors should be avoided.

According to the theory of "technology S-curves", because the space for performance improvement of one technology is limited, the benefit of the investment into a mature technology must be weighed against the potential of an emerging technology [8]. Conversely, the principle feasibility of new process chains also does not represent that the chains are always worth being realized in respect of the economics. For this, defining judgement criterion with consideration of the potential for application of the new building or the reforming process chains is an issue which must be faced.

\section{Conclusion}

Loopless Production suggests a way to overcome errors in manufacturing through rationally selecting and coordinating processes rather than just by using closed loops. This requires that materials should be processed in accordance with their specific properties. Unknown errors as unforeseeable deviations will be caused due to the insufficient understanding of process functional mechanisms or by the imperfect process fulfillments. Systematic errors as foreseeable deviations will be caused due to improper process applications. Process Signatures helps identify the errors through its approach for detailed analyses of mechanisms of energy-material interactions. The combination of these two ideas shall allow the simplification of the work for the rationalization of process sequences, the streamlining of closed loops as well as the selection of optimal substitute processes.

\section{Acknowledgements}

This work was supported by the German Research Foundation (DFG) for funding the subproject F07-Processes with thermochemical impact of the Transregional Collaborative Research Center 136 (CRC/TRR 136).

\section{References}

[1] Jawahir, I.S., Brinksmeier, E., M’saoubi, R., Aspinwall, D.K., Outeiro, J.C., Meyer, D., Umbrello, D. and Jayal, A.D. (2011) Surface Integrity in Material Removal Processes: Recent advances. CIRP Annals-Manufacturing Technology, 60, 603-626.

[2] Van Ravenswaaij, R., Van Tijum, R., Hora, P., Van Den Boogaard, T. and Engel, U. (2013) Towards Zero-Defect Manufacturing of Smal Metal Parts. IDDRG 2013 Conference Proceedings, Zurich, 87-92. http://www.megafit-project.eu

[3] Teti, R. (2011) Intelligent Fault Correction and self Optimizing Manufacturing Systems. Intelligent Fault Correction and self Optimizing Manufacturing systems. http://www.ifacom.org

[4] Mansori, M.E., Mezghani, S., Sabri, L. and Zahouani, H. (2010) On Concept of Process Signature in Analysis of Multistage Surface Formation. Surface Engineering, 
26, 216-223.

[5] Brinksmeier, E., Gläbe, R., Klocke, F. and Lucca, D.A. (2011) Process Signatures-An Alternative Approach to Predicting Functional Workpiece Properties. Procedia Engineering, 19, 44-52.

[6] Brinksmeier, E., Klocke, F., Lucca, D.A., Sölter, J. and Meyer, D. (2014) Process Signatures-A New Approach to Solve the Inverse Surface Integrity Problem in Machining Processes. Procedia CIRP, 13, 429-434.

[7] Zhao, T., Mehrafsun, S. and Vollertsen, F. (2016) November Energy-Based Approach as an Example for a Process Signature for Laser Microprocessing. In SPIE/COS Photonics Asia, 100180D-100180D.

[8] Schilling, M.A. and Esmundo, M. (2009) Technology S-Curves in Renewable Energy Alternatives: Analysis and Implications for Industry and Government. Energy Policy, 37, 1767-1781.

[9] Pirati, A., Peeters, R., Smith, D., Lok, S., van Noordenburg, M., van Es, R., Verhoeven, E., Meijer, H., Minnaert, A., van der Horst, J.W. and Meiling, H. (2016) March. EUV Lithography Performance for Manufacturing: Status and Outlook. In SPIE Advanced Lithography, 97760A-97760A.

[10] Vollertsen F. (2016) Loopless Production: Definition and Examples from Joining. 69th IIW Annual Assembly and International Conference, Melbourne Convention Exhibition Centre, Melbourne, 10-15.

\section{Submit or recommend next manuscript to SCIRP and we will provide best service for you:}

Accepting pre-submission inquiries through Email, Facebook, LinkedIn, Twitter, etc. A wide selection of journals (inclusive of 9 subjects, more than 200 journals) Providing 24-hour high-quality service User-friendly online submission system Fair and swift peer-review system Efficient typesetting and proofreading procedure Display of the result of downloads and visits, as well as the number of cited articles Maximum dissemination of your research work

Submit your manuscript at: http://papersubmission.scirp.org/

Or contact eng@scirp.org 